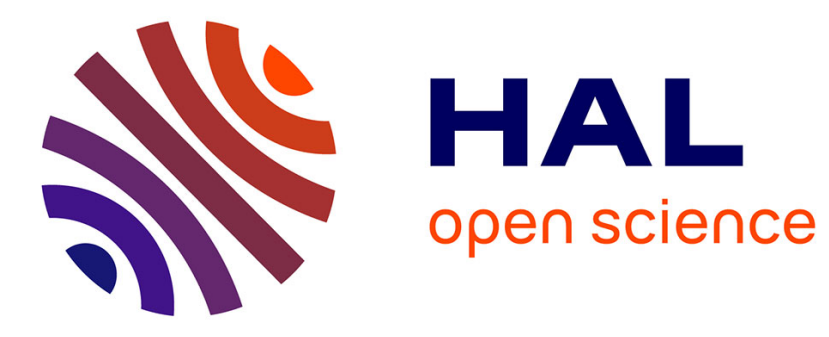

\title{
Mine 'Em All: A Note on Mining All Graphs
}

\author{
Ondřej Kuželka, Jan Ramon
}

\section{To cite this version:}

Ondřej Kuželka, Jan Ramon. Mine 'Em All: A Note on Mining All Graphs. 25th International Conference on Inductive Logic Programming, Katsumi Inoue, Hayato Ohwada, Akihiro Yamamoto, Aug 2015, Kyoto, Japan. hal-01321448

\section{HAL Id: hal-01321448 https://hal.science/hal-01321448}

Submitted on 25 May 2016

HAL is a multi-disciplinary open access archive for the deposit and dissemination of scientific research documents, whether they are published or not. The documents may come from teaching and research institutions in France or abroad, or from public or private research centers.
L'archive ouverte pluridisciplinaire HAL, est destinée au dépôt et à la diffusion de documents scientifiques de niveau recherche, publiés ou non, émanant des établissements d'enseignement et de recherche français ou étrangers, des laboratoires publics ou privés. 


\title{
Mine 'Em All: A Note on Mining All Graphs
}

\author{
Ondřej Kuželka ${ }^{1}$ and Jan Ramon ${ }^{2,3}$ \\ 1 School of Computer Science \& Informatics, Cardiff University, UK \\ Kuzelka0@cardiff.ac.uk \\ 2 Department of Computer Science, KU Leuven, Belgium \\ 3 INRIA, Lille, France \\ jan.ramon@cs.kuleuven. be
}

\begin{abstract}
We study the complexity of the problem of enumerating all graphs with frequency at least 1 and computing their support. We show that there are hereditary classes of graphs for which the complexity of this problem depends on the order in which the graphs should be enumerated (e.g. from frequent to infrequent or from small to large). For instance, the problem can be solved with polynomial delay for databases of planar graphs when the enumerated graphs should be output from large to small but it cannot be solved even in incremental-polynomial time when the enumerated graphs should be output from most frequent to least frequent (unless $\mathrm{P}=\mathrm{NP}$ ).
\end{abstract}

\section{Introduction}

In this paper we study graph mining problems from a nontraditional perspective. We are inspired by the question which properties of the problem make some graph mining problems solvable in incremental polynomial time or with polynomial delay. Here, we do not require the discovered graph patterns to be frequent and we want to output all patterns occurring in at least one database graph. However, we still want to also output their occurrences. In addition, we constrain the order in which the patterns should be printed, e.g. from most frequent patterns to least frequent patterns, which allows us to connect our results to results on (in)frequent graph mining. Surprisingly, for several graph classes, we show that different orders lead to very different computational complexities. For instance mining planar graphs cannot be done in incremental-polynomial time when the output graphs should be ordered by frequency but it can be done with polynomial delay when they should be ordered from largest to smallest.

\section{Preliminaries}

In this section we first briefly review some basic concepts and fix the notations used in this paper. We start with some standard definitions from graph theory. 
Graphs. An undirected graph is a pair $(V, E)$, where $V$ is a finite set of vertices and $E \subseteq\{e \subseteq V:|e|=2\}$ is a set of edges. Two vertices are said to be adjacent (in $G$ ) if they are connected by an edge (of the graph $G$ ). A labeled undirected graph is a triple $(V, E, \lambda)$, where $(V, E)$ is an undirected graph and $\lambda: V \cup E \rightarrow \Sigma$ is a function assigning a label from an alphabet $\Sigma$ to every element of $V \cup E$. We will denote the set of vertices, the set of edges, and the labeling function of a graph $G$ by $V(G), E(G)$, and $\lambda_{G}$, respectively. We define $|G|=|V(G)|+|E(G)|$ and call this the order of $G$. Note that in graph theory, often other notions of 'order' are used, measuring only the number of edges or the number of vertices. A graph $G^{\prime}$ is a subgraph of a graph $G$, if $V\left(G^{\prime}\right) \subseteq V(G), E\left(G^{\prime}\right) \subseteq E(G)$, and $\lambda_{G^{\prime}}(x)=\lambda_{G}(x)$ for every $x \in V\left(G^{\prime}\right) \cup E\left(G^{\prime}\right) ; G^{\prime}$ is an induced subgraph of $G$ if it is a subgraph of $G$ satisfying $\{u, v\} \in E\left(G^{\prime}\right)$ if and only if $\{u, v\} \in E(G)$ for every $u, v \in V\left(G^{\prime}\right)$. For a subset $S \subseteq V(G), G[S]$ denotes the (unique) induced subgraph of $G$ with vertex set $S$. A contraction of an edge $e=\{u, v\}$ in a graph $G$ is an operation which produces a new graph by replacing $u$ and $v$ in $V(G)$ as well as in all $\{x, y\} \in E(G)$ by a new vertex $w$ (pictorially, this can be imagined as shrinking the edge). A subdivision of an edge $e=\{u, v\}$ is an operation which produces a new graph by removing $e$ and adding a path connecting $u$ and $v$.

Tree decomposition, tree-width. The notion of tree-width was reintroduced in $[11,3]$. It proved to be a useful parameter of graphs in algorithmic graph theory. A tree-decomposition of a graph $G$, denoted $T D(G)$, is a pair $(T, \mathcal{X})$, where $T$ is a rooted unordered tree and $\mathcal{X}=\left(X_{z}\right)_{z \in V(T)}$ is a family of subsets of $V(G)$ satisfying

(i) $\cup_{z \in V(T)} X_{z}=V(G)$,

(ii) for every $\{u, v\} \in E(G)$, there is a $z \in V(T)$ such that $u, v \in X_{z}$, and

(iii) $X_{z_{1}} \cap X_{z_{3}} \subseteq X_{z_{2}}$ for every $z_{1}, z_{2}, z_{3} \in V(T)$ such that $z_{2}$ is on the simple path connecting $z_{1}$ with $z_{3}$ in $T$.

The set $X_{z}$ associated with a node $z$ of $T$ is called the $b a g$ of $z$. The nodes of $T$ will often be referred to as the nodes of $T D(G)$. The tree-width of $T D(G)$ is $\max _{z \in V(T)}\left|X_{z}\right|-1$, and the tree-width of $G$, denoted $\operatorname{tw}(G)$, is the minimum tree-width over all tree-decompositions of $G$. By graphs of bounded tree-width we mean graphs of tree-width at most $k$, where $k$ is some constant.

A class of graphs $\mathcal{G}$ is called hereditary if for any graph $G \in \mathcal{G}$ all its subgraphs also belong to $\mathcal{G}$. The class of graphs of treewidth at most $k$ is hereditary. The same also holds for planar graphs (as clearly any subgraph of a planar graph is still planar).

Graph isomorphism, Graph canonization. Graphs $G$ and $G^{\prime}$ are isomorphic if there exists a bijection $\pi: V(G) \rightarrow V\left(G^{\prime}\right)$ such that $\{u, v\} \in E(G)$ if and only if $\{\pi(u), \pi(v)\} \in E\left(G^{\prime}\right)$. Graph canonization is a function from graphs to strings such that two graphs have the same canonization if and only if they are isomorphic. 
Subgraph isomorphism and induced subgraph isomorphism. We say that a graph $G_{1}$ is subgraph isomorphic to a graph $G_{2}$ if $G_{1}$ is isomorphic to a subgraph of $G_{2}$. We say that a graph $G_{1}$ is induced subgraph isomorphic to a graph $G_{2}$ if $G_{1}$ is isomorphic to an induced subgraph of $G_{2}$. Deciding whether a graph is (induced) subgraph isomorphic to another graph is NP-complete and it remains NP-complete even for bounded-treewidth graphs [10]. Note that there are graph classes, e.g. bounded-treewidth graphs or planar graphs, for which isomorphism can be decided in polynomial time but for which subgraph isomorphism is NPcomplete. We are mostly interested in such classes because for them it is not obvious whether fast graph mining algorithms exist.

Homeomorphism and induced homeomorphism. We say that a graph $G_{1}$ is homeomorphic to a graph $G_{2}$ if there is a graph $G_{1}^{\prime}$ which can be obtained from $G_{1}$ by subdividing its edges and $G_{1}^{\prime}$ is subgraph isomorphic to $G_{2}$. We say that a graph $G_{1}$ is induced homeomorphic to a graph $G_{2}$ if there is a graph $G_{1}^{\prime}$ which can be obtained from $G_{1}$ by subdividing its edges and $G_{1}^{\prime}$ is induced subgraph isomorphic to $G_{2}$. Deciding if a graph is homeomorphic to another graph is NP-complete even for graphs of bounded treewidth and unbounded maximum degree [10].

Minor embedding and induced minor embedding. We say that a graph $G_{1}$ is minor-embeddable to a graph $G_{2}$ if there is a graph $G_{1}^{\prime}$ isomorphic to $G_{1}$ which can be obtained from a subgraph of $G_{2}$ by contracting edges and deleting loops and multiple-edges thus produced. We say that a graph $G_{1}$ is induced minorembeddable to a graph $G_{2}$ if there is a graph $G_{1}^{\prime}$ isomorphic to $G_{1}$ which can be obtained from an induced subgraph of $G_{2}$ by contracting edges and deleting loops and multiple-edges thus produced. Deciding if a graph is minor-embeddable to another graph is NP-complete even for graphs of bounded treewidth and unbounded maximum degree [10].

Fixed-parameter tractability. Formally, a parameterized decision problem is a language $L \subseteq \Sigma^{*} \times N$ where $\Sigma$ is a finite alphabet and $N$ is the set of natural numbers [1]. An instance of a parametrized problem is a pair $(x, k)$ where $k \in N$ is called parameter of the problem. A problem is fixed-parameter tractable (abbreviated $F P T$ ) if there exists an algorithm for solving instances of it which runs in time $|x|^{O(1)} \cdot f(k)$ where $f$ is a computable function. Notice that whether a problem is fixed-parameter tractable depends on the selected parameterization. For instance, when the parameter of the problem is $|x|$, i.e. the actual size of the problem, then any problem e.g. from classes such as e.g. NP, EXP, NEXP is fixed-parameter tractable with such a parameterization. On the other hand, it is widely believed that e.g. the clique problem is not fixed-parameter tractable with the parameter being size of the clique. To capture a conjectured intractability hierarchy, the W-hierarchy is used which consists of an infinite number of increasingly more intractable classes $\mathrm{W}[1], \mathrm{W}[2]$, etc. The W-hierarchy is based on fixed-parameter reductions. A problem $L_{A}$ is fixed-parameter reducible to a 
problem $L_{B}$ if there exists an algorithm for transforming instances $(x, k)_{A}$ of the problem $L_{A}$ to instances $\left(x^{\prime}, k^{\prime}\right)_{B}$ of the problem $B$ such that:

(i) the transformation algorithm runs in time $|x|^{O(1)} \cdot f(k)$ where $f$ is a computable function,

(ii) $k^{\prime} \leq g(k)$ where $g$ is a computable function,

(iii) $(x, k)_{A} \in L_{A}$ if and only if $\left(x^{\prime}, k^{\prime}\right)_{B} \in L_{B}$ (informally, $(x, k)_{A}$ has a 'yes' solution if and only if $\left(x^{\prime}, k^{\prime}\right)_{B}$ has a 'yes' solution).

\section{Graph Mining Problems}

In this section, we define the mining problems studied in this paper and describe their basic properties. We start with the definition of the classical frequent connected graph mining problem.

A transaction database is a multiset of graphs from a given class $\mathcal{G}$. Given a pattern matching operator $\preccurlyeq$ (subgraph isomorphism or induced subgraph isomorphism), the frequency of a graph $G$ in a transaction database $D B$, denoted by freq $(G, D B)$, is given as freq $(G, D B)=\left|\left\{G^{\prime} \in D B \mid G \preccurlyeq G^{\prime}\right\}\right|$. Given a threshold $t, G$ is said to be frequent if freq $(G, D B) \geq t$. The elements of the multiset $\left\{G^{\prime} \in D B \mid G \preccurlyeq G^{\prime}\right\}$ are called occurrences of the graph $G$ in the database $D B$. We will often represent the set of occurrences also just by names or IDs of the graphs contained in it (e.g. $G_{1}$ will be represented just by "1").

Definition 1 (The Frequent Connected Graph Mining (FCGM) ProbLEM). Given a class $\mathcal{G}$ of graphs, a transaction database $D B$ of graphs from $\mathcal{G}$, a pattern matching operator $\preccurlyeq$, and frequency threshold, list the set of frequent connected graphs $G \in \mathcal{G}$ and their occurrences.

In this paper, we are interested in another closely related type of problem which is to mine all graphs with frequency at least one in certain order.

Definition 2 (The Ordered Mining Problems). Given a class $\mathcal{G}$ of graphs, a transaction database $D B$ of graphs from $\mathcal{G}$ and a pattern matching operator $\preccurlyeq$, list the set of connected graphs $G \in \mathcal{G}$ with freq $(G, D B) \geq 1$ and their occurrences in the transactions in the given order ${ }^{4}$ :

- from most frequent to least frequent ( $\mathrm{ALL}_{F \rightarrow I}$ problem),

- from least frequent to most frequent $\left(\mathrm{ALL}_{I \rightarrow F}\right.$ problem),

- from smallest size to largest size ( $\mathrm{ALL}_{S \rightarrow L}$ problem),

- from largest size to smallest size ( $\mathrm{ALL}_{L \rightarrow S}$ problem).

Here size of a graph $G$ refers to $|E(G)|$ when $\preccurlyeq$ is subgraph isomorphism and to $|V(G)|$ when $\preccurlyeq$ is induced subgraph isomorphism.

\footnotetext{
${ }^{4} \mathrm{ALL}_{F \rightarrow I}$ stands for 'frequent to infrequent', $\mathrm{ALL}_{I \rightarrow F}$ stands for 'infrequent to fre-
} quent', $\mathrm{ALL}_{S \rightarrow L}$ stands for 'small to large' and $\mathrm{ALL}_{L \rightarrow S}$ stands for 'large to small'. 
The parameter of the above problems is the size of $D B$. One can easily construct examples for which the number of frequent connected subgraphs is exponential in this parameter. Thus, in general, the set of all frequent connected subgraphs cannot be computed in time polynomial only in the size of $D B$. Since this is a common feature of many listing problems, the following problem classes are usually considered in the literature (see, e.g., [6]). For some input $I$, let $O$ be the output set of some finite cardinality $N$. Then the elements of $O$, say $o_{1}, \ldots, o_{N}$, are listed with:

- polynomial delay if the time before printing $o_{1}$, the time between printing $o_{i}$ and $o_{i+1}$ for every $i=1, \ldots, N-1$, and the time between printing $o_{N}$ and the termination is bounded by a polynomial of the size of $I$,

- incremental polynomial time if $o_{1}$ is printed with polynomial delay, the time between printing $o_{i}$ and $o_{i+1}$ for every $i=1, \ldots, N-1$ (resp. the time between printing $o_{N}$ and the termination) is bounded by a polynomial of the combined size of $I$ and the set $\left\{o_{1}, \ldots, o_{i}\right\}$ (resp. $O$ ),

- output polynomial time (or polynomial total time) if $O$ is printed in time polynomial in the combined size of $I$ and the entire output $O$.

Clearly, polynomial delay implies incremental polynomial time, which, in turn, implies output polynomial time. Furthermore, in contrast to incremental polynomial time, the delay of an output polynomial time algorithm may be exponential in the size of the input even before printing the first element of the output.

Example 1. Let us have graphs $G_{1}=(\{1,2,3\},\{\{1,2\},\{2,3\},\{3,1\}\})$ and $G_{2}=$ $(\{1,2,3,4\},\{\{1,2\},\{2,3\},\{3,4\}\}), D B=\left\{G_{1}, G_{2}\right\}$ and let $t=2$. A solution of the FCGM problem is

$$
\begin{aligned}
& H_{1}=(\{1\},\{\}), O C C_{1}=\{1,2\} \\
& H_{2}=(\{1,2\},\{\{1,2\}\}), O C C_{2}=\{1,2\} \\
& H_{3}=(\{1,2,3\},\{\{1,2\},\{2,3\}\}), O C C_{3}=\{1,2\}
\end{aligned}
$$

where $O C C_{i}$ denotes the occurrences of the graph $H_{i}$. A solution of the problem $\mathrm{ALL}_{F \rightarrow I}$ is

$$
\begin{aligned}
& H_{1}=(\{1\},\{\}), O C C_{1}=\{1,2\} \\
& H_{2}=(\{1,2\},\{\{1,2\}\}), O C C_{2}=\{1,2\} \\
& H_{3}=(\{1,2,3\},\{\{1,2\},\{2,3\}\}), O C C_{3}=\{1,2\} \\
& H_{4}=(\{1,2,3\},\{\{1,2\},\{2,3\},\{3,1\}\}), O C C_{4}=\{1\} \\
& H_{5}=(\{1,2,3,4\},\{\{1,2\},\{2,3\},\{3,4\}\}), O C C_{4}=\{2\}
\end{aligned}
$$

Remark 1. There is an incremental-polynomial-time algorithm for the FCGM (FCIGM) problem if and only if there is an incremental-polynomial time algorithm for $\mathrm{ALL}_{F \rightarrow I}$ with (induced) subgraph isomorphism as a pattern matching operator. 
In general, we will see in the next sections that the different ordered mining problems possess different computational complexities under standard complexity theoretic assumptions.

\section{Mining All (Induced) Subgraphs}

\subsection{Negative Results}

In this section, we provide several negative results regarding complexity of some of the enumeration problems considered in this paper. The first theorem connects the hardness of the frequent subgraph enumeration problem to fixed-parameter tractability of the pattern matching operator (subgraph isomorphism or induced subgraph isomorphism).

Theorem 1. Let $\mathcal{G}$ be a class of graphs. Let $\preccurlyeq$ be either subgraph isomorphism or induced subgraph isomorphism. If deciding $H \preccurlyeq G$ where $G, H \in \mathcal{G}$ is not fixedparameter tractable with the parameter $|H|$ then there is no output-polynomialtime algorithm for enumerating frequent connected graphs from databases consisting of graphs from $\mathcal{G}$.

Proof. Let us suppose that there is an algorithm for mining frequent connected graphs from databases of graphs from $\mathcal{G}$ which runs in output-polynomial time. Let $G, H \in \mathcal{G}$. We show that then it is always possible to decide whether $H \preccurlyeq G$ holds in time $f(|H|) \cdot|G|^{O(1)}$. If $|H|>|G|$ then $H \npreceq G$ and we can finish. If $|H| \leq|G|$, we set $D B=\{H, G\}$ and we let the pattern mining algorithm run on $D B$ with minimum frequency $t=2$. Since there are at most $2^{|E(H)|}$ connected subgraphs of $H$, the mining algorithm will produce an output of length at most $|H| \cdot 2^{|E(H)|}$ in time poly $\left(|H|+|G|,|H| \cdot 2^{|E(H)|}\right)$. If the output contains a frequent graph $F$ such that $|V(F)|=|V(H)|$ and $|E(F)|=|E(H)|$ (such a graph $F$ must be isomorphic to $H$ ), we return true. Otherwise, we return false. Now, if we return true then $H \preccurlyeq G$ must hold because if a graph $F$ isomorphic to $H$ is frequent, it must hold $F \preccurlyeq G$ and therefore also $H \preccurlyeq G$. Similarly, if we return false then there is no frequent graph $F$ isomorphic to $H$ and therefore $H \npreceq G$. This all put together runs in time $f(|H|) \cdot|G|^{O(1)}$ where $f$ is a computable function. However, then the just described procedure would give us an algorithm for the pattern matching operator which would be fixed-parameter tractable with the parameter $|H|$ which is a contradiction.

From the proof of the above theorem, we can obtain the following corollary ${ }^{5}$.

${ }^{5}$ The hardness for the $\mathrm{ALL}_{F \rightarrow I}$ problem follows from Theorem 1 together with Remark 1 , whereas hardness of the $\mathrm{ALL}_{S \rightarrow L}$ problem follows from a simple modification of the proof of Theorem 1 where we use a hypothetic incr.-poly.-time algorithm for solving the $\mathrm{ALL}_{S \rightarrow L}$ problem and stop it after printing the first graph with more edges than $H$ (or more vertices than $H$ in the case of induced subgraph mining). 
Corollary 1. Let $\mathcal{G}$ be a class of graphs. Let $\preccurlyeq$ be either subgraph isomorphism or induced subgraph isomorphism. If deciding $H \preccurlyeq G$ where $G, H \in \mathcal{G}$ is not fixedparameter tractable with the parameter $|H|$ then $\mathrm{ALL}_{F \rightarrow I}$ and $\mathrm{ALL}_{S \rightarrow L}$ cannot be solved in incremental polynomial time.

However, there are also graph classes with FPT subgraph isomorphism, e.g. planar graphs [9], for which $\mathrm{ALL}_{F \rightarrow I}$ cannot be solved in incr.-poly. time ${ }^{6}$.

Theorem 2. The problem $\mathrm{ALL}_{F \rightarrow I}$ cannot be solved in incremental polynomial time for the class $\mathcal{G}$ of planar graphs and subgraph isomorphism as pattern matching operator.

Proof. We can use NP-hardness of Hamiltonian cycle problem [2], similarly as [5]. We construct a database consisting of a given graph $G$ and a cycle $C$ on $|V(G)|$ vertices. A graph isomorphic to $C$ with frequency 2 (recall that frequency is given implicitly by the printed occurrences) is output by the mining algorithm among the first $|V(G)|+1$ graphs if and only if $G$ contains a Hamiltonian cycle. It is easy to see that we could then use an incremental-polynomial time algorithm for the $\mathrm{ALL}_{F \rightarrow I}$ problem to solve the Hamitonian cycle problem. Therefore there is no incremental-polynomial time algorithm for the $\mathrm{ALL}_{F \rightarrow I}$ problem (unless $\mathrm{P}=\mathrm{NP})$.

This theorem is interesting because in Section 4.2, we will see that the problem $\mathrm{ALL}_{L \rightarrow S}$ can be solved with polynomial delay for planar graphs.

Even stronger negative result can be obtained for the problem $\operatorname{ALL}_{I \rightarrow F}$.

Theorem 3. Let $\mathcal{G}$ be a class of graphs. Let $\preccurlyeq$ be either subgraph isomorphism or induced subgraph isomorphism. If deciding $H \preccurlyeq G$ where $G, H \in \mathcal{G}$ is NP-hard then $\mathrm{ALL}_{I \rightarrow F}$ cannot be solved in incremental polynomial time (unless $\mathrm{P}=\mathrm{NP}$ ).

Proof. Let $H$ and $G$ be graphs from $\mathcal{G}$. We will show how to use an incrementalpolynomial-time algorithm for the problem $\operatorname{ALL}_{I \rightarrow F}$ to decide whether $H \preccurlyeq G$ in polynomial time. We construct a database of graphs $D B=\{H, G, G\}$ and let the algorithm for the problem $\mathrm{ALL}_{I \rightarrow F}$ run until it outputs a graph and its occurrences (implicitly giving us also the frequency) and then we stop it (it follows from definition of incremental-polynomial time that this will run only for time polynomial in the sizes of $H$ and $G$ ). It is easy to see that the output graph has frequency 1 if and only if $H \npreceq G$. Thus, if the frequency of the output graph is 1 we return 'not (induced) subgraph isomorphic' and if the frequency of the output graph is greater than 1 then we return '(induced) subgraph isomorphic'. Therefore if deciding $H \preccurlyeq G$ is NP-hard for graphs from $\mathcal{G}$ there cannot be an incremental-polynomial-time algorithm for the problem $\mathrm{ALL}_{I \rightarrow F}$ (unless $\mathrm{P}=$ NP).

Using the fact that (induced) subgraph isomorphism is NP-complete even for bounded-treewidth graphs [10] and planar graphs [9], we can obtain the following.

${ }^{6}$ The complexity of the $\mathrm{ALL}_{S \rightarrow L}$ problem in these cases remains an interesting open problem. 
Corollary 2. The problem $\mathrm{ALL}_{I \rightarrow F}$ cannot be solved in incremental polynomial time for the class of planar graphs and for the class of bounded-treewidth graphs.

Note that Theorem 1 cannot be made as strong as Theorem 3 (i.e. showing that $\mathrm{ALL}_{F \rightarrow I}$ cannot be solved in incremental-polynomial time if the pattern matching operator is NP-hard) because the results of Horváth and Ramon from [5] demonstrate that even if the pattern matching operator is NP-hard there can be an incremental-polynomial-time algorithm for mining frequent subgraphs. Theorem 3 shows that we cannot expect such a result for mining infrequent subgraphs (i.e. subgraphs with frequency below a threshold).

\subsection{Positive Results for $\mathrm{ALL}_{F \rightarrow I}$ and $\mathrm{ALL}_{S \rightarrow L}$}

Before presenting our new results for $\mathrm{ALL}_{L \rightarrow S}$ in the next section, we note that there exists the following positive result for frequent graph mining from boundedtreewidth graphs, which was presented in $[4,5]$.

Theorem 4 (Horváth and Ramon [5], Horváth, Otaki and Ramon [4]). The FCGM and FCIGM problems can be solved in incremental-polynomial time for the class of bounded-treewidth graphs.

This result directly translates to a positive result for the problem $\mathrm{ALL}_{F \rightarrow I}$ summarized in the following corollary (recall that we have shown in the previous section that $\mathrm{ALL}_{I \rightarrow F}$ cannot be solved in incremental-polynomial time for bounded-tree-width graphs) and to a result for the problem $\operatorname{ALL}_{S \rightarrow L}$ (this other result follows from the fact that the respective algorithms are level-wise).

Corollary 3. The problems $\mathrm{ALL}_{F \rightarrow I}$ and $\mathrm{ALL}_{S \rightarrow L}$ can be solved in incrementalpolynomial time for the class of bounded-treewidth graphs.

\subsection{Positive Results for $\mathrm{ALL}_{L \rightarrow S}$}

In this section, we describe an algorithm called LARGERToSmaller (Alg. 1) which, when given a class of graphs $\mathcal{G}$ in which isomorphism can be decided in polynomial time, solves the problem $\mathrm{ALL}_{L \rightarrow S}$ in incremental-polynomial time, or with polynomial delay if $\mathcal{G}$ also admits a polynomial-time canonization. The main employed trick is the observation that for the problem $\operatorname{ALL}_{L \rightarrow S}$ it is not necessary to use subgraph isomorphism explicitly for computing occurrences.

The algorithm maintains a data structure $A L L$ storing key-value pairs where keys are graphs and values are sets of $\operatorname{IDs}^{7}$ of graphs in which the given key graph is contained either as a subgraph or as an induced subgraph (depending on whether we are mining subgraphs or induced subgraphs). The data structure provides four functions/procedures: $\operatorname{ADD}(K, O C C, A L L), \mathrm{GET}(K, A L L)$, $\operatorname{KEYS}(n, A L L)$, and $\operatorname{DELETE}(n, A L L)$.

\footnotetext{
${ }^{7}$ Here, IDs are just some identifiers given to the database graphs.
} 
The procedure $\operatorname{ADD}(K, O C C, A L L)$ adds the IDs contained in $O C C$ to the set associated with a key contained in $A L L$ which is isomorphic to $K$ or, if no such key is contained in $A L L$, the procedure stores $K$ in $A L L$ and associates $O C C$ with it. If we restrict attention to graphs from a class $\mathcal{G}$ for which a polynomial-time canonization function running in $O(p(|H|))$ exists (where $p$ is a polynomial) then the procedure $\operatorname{ADD}(K, O C C, A L L)$ can be implemented to run in time $O(p(|K|)$ ) (we can just store the key graphs as canonical strings, therefore a hashtable with constant-time methods for finding and adding values by their keys can be used). If a polynomial-time canonization function does not exist but graph isomorphism can be decided in time $O\left(p_{\text {iso }}(|K|)\right)$ where $p_{\text {iso }}$ is a polynomial then the procedure $\operatorname{ADD}(K, O C C, A L L)$ can be implemented to run in time $O\left(\mid\left\{K^{\prime} \in K E Y S(A L L):\left|V\left(K^{\prime}\right)\right|=|V(K)|\right.\right.$ and $\left.\left|E\left(K^{\prime}\right)\right|=|E(K)|\right\} \mid$. $\left.p_{\text {iso }}(|K|)\right)$.

The function $\mathrm{GET}(K, A L L)$ returns all IDs associated with a key isomorphic to $K$. The exactly same considerations as for the procedure ADD apply also for this function.

The function $\operatorname{KEYS}(n, A L L)$ returns a pointer to a linked list containing all key graphs stored in $A L L$ which have size $n$. Since the data structure $A L L$ does not allow deletion of individual keys, it is easy to maintain such a linked list ${ }^{8}$.

Finally, the procedure $\operatorname{DELETE}(n, A L L)$ removes the pointer ${ }^{9}$ to the linked list containing all key graphs of order $n$ stored in $A L L$.

The algorithm LARGERTOSMALLER fills in the data structure $A L L$, starting with the largest graphs and proceeding to the smaller ones. When it processes a graph $H$, it first prints it and the IDs of the graphs associated to it in the data structure $A L L$, and then it calls the function REFINE which returns all connected subgraphs $H^{\prime}$ of $H$ which can be obtained from $H$ by removing an edge or an edge and its incedent vertex of degree one, in the case of subgraph mining, or just by removing a vertex and all its incident edges, in the case of induced subgraph mining. It then associates all occurrences of the graph $H$ with the graphs $H^{\prime}$ in the datastructure $A L L$ using the procedure ADD. Since the same graph $H^{\prime}$ may be produced from different graphs $H$, the occurrences of $H^{\prime}$ accumulate and we can prove that when a graph $H$ is printed, the data structure $A L L$ already contains all IDs of graphs in which $H$ is contained.

Theorem 5. Let $\mathcal{G}$ be a hereditary class of graphs with isomorphism decidable in polynomial time. Given a database $D B$ of graphs from $\mathcal{G}$, the algorithm LARGERTOSMALLER solves the problem $\mathrm{ALL}_{L \rightarrow S}$ in incremental polynomial time. If the graphs from $\mathcal{G}$ also admit a poly-time canonization then the algorithm LARGERTOSMALLER solves the problem $\mathrm{ALL}_{L \rightarrow S}$ with polynomial delay.

\footnotetext{
8 The reason why the function KEYS does not just return all the key graphs but rather a pointer to the linked list is that if it did otherwise, Algorithm 1 could never run with polynomial delay

${ }^{9}$ Note that we just remove the pointer and do not actually "free" the memory occupied by the graphs. For the practical implementation, we used a programming language with a garbage collector.
} 


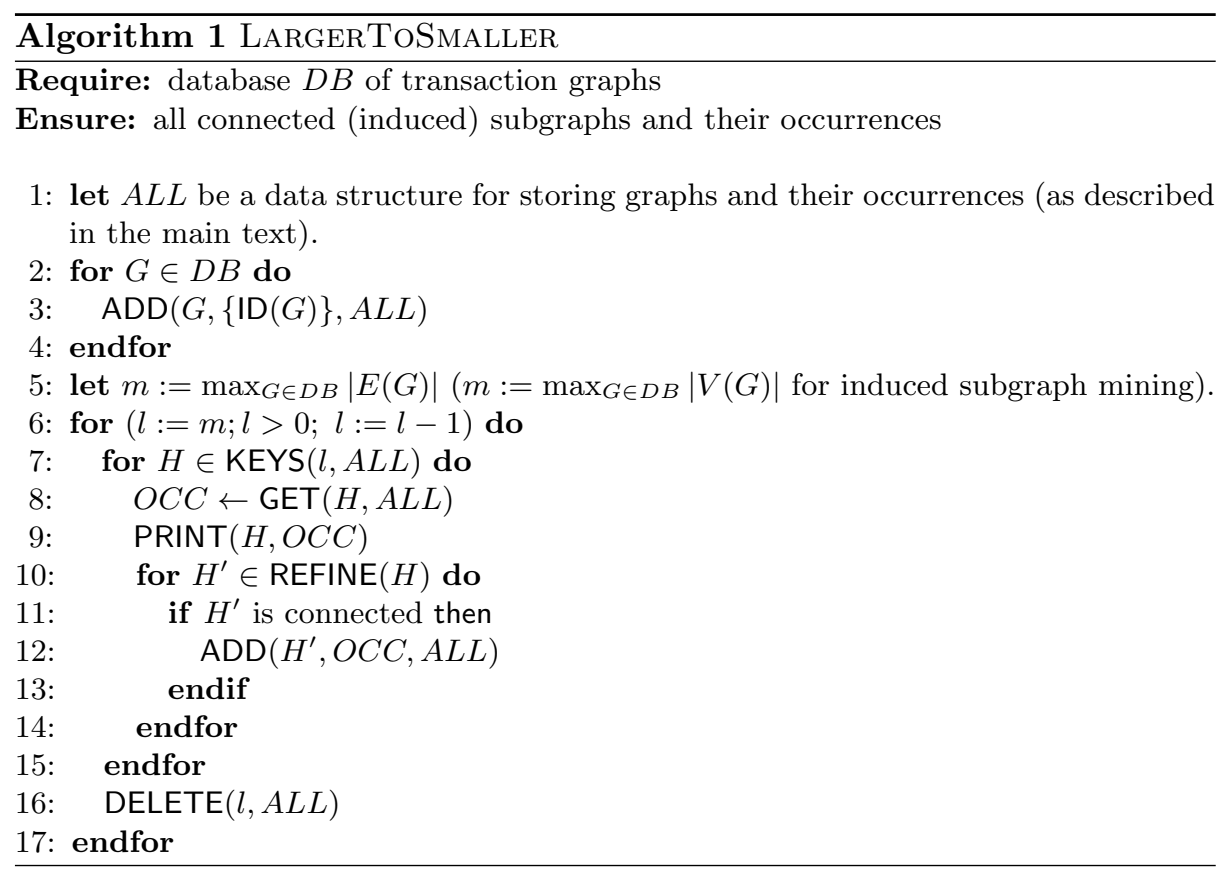

Proof. First, we show that the algorithm prints every (induced) subgraph of the graphs in $D B$. Let us assume, for contradiction, that this is not the case and let $G^{*}$ be a maximal connected graph which is a (induced) subgraph of a graph in the database and such that no graph isomorphic to it is printed by the algorithm. It is easy to verify that such a graph $G^{*}$ cannot be isomorphic to any graph in $D B$. Since $G^{*}$ is not isomorphic to a graph from $D B$ and since it is a maximal graph not printed, there must be a supergraph $G^{\prime}$ of $G^{*}$ such that $\left|E\left(G^{*}\right)\right|+1=\left|E\left(G^{\prime}\right)\right|$ in the case of subgraph isomorphism $\left(\left|V\left(G^{*}\right)\right|+1=\left|V\left(G^{\prime}\right)\right|\right.$ in the case of induced subgraph isomorphism, respectively) and such that a graph isomorphic to it is printed by the algorithm. However, if such a graph was printed then a graph isomorphic to $G^{*}$ would have to be in $\operatorname{REFINE}\left(G^{\prime}\right)$ and would have to be printed eventually, which is a contradiction.

Second, we show that the occurrences printed with each graph are correct. First, if a printed graph $G$ does not have any strict supergraph in the database then it must be equivalent to one or more database graphs. However, it is easy to check by simple inspection of the algorithm that the occurrences must be correct in this case. For the rest of the printed graphs (i.e. graphs which have strict supergraphs in the databse), let us assume, for contradiction, that $G^{*}$ is a maximal graph printed by the algorithm for which the printed occurrences are not correct, i.e. either there is an ID of a database graph printed for $G^{*}$ of which $G^{*}$ is not an (induced) subgraph or there is an ID of a database graph not printed for $G^{*}$ of which $G^{*}$ is actually an (induced) subgraph. (False occurrence:) If there is an ID of a database graph of which $G^{*}$ is not a (induced) subgraph then at 
least one of the graphs from which $G^{*}$ can be obtained by refinement must have an ID associated which it should not have. But then $G^{*}$ could not be maximal graph with this property, which is a contradiction. (Missing occurrence:) If there is a missing ID of a database graph of which $G^{*}$ is a (induced) subgraph then one of the following must be true: (i) $G^{*}$ is isomorphic to the database graph but then it is easily seen by inspection of the algorithm that the ID of this graph cannot be missing from the occurrences of $G^{*}$, (ii) there is a strict supergraph $G^{\prime}$ of $G^{*}$ which is (induced) subgraph isomorphic to the database graph and which is not printed (and therefore its occurrences are not added to the data structure ALL), but this is not possible as the first part of the proof shows, (iii) there is a strict supergraph $G^{\prime}$ of $G^{*}$ which is (induced) subgraph isomorphic to the database graph and the respective ID was not associated to it, but then $G^{*}$ could not be a maximal graph with this property. Thus, we have a contradiction.

Third, we show that if there is a polynomial-time isomorphism algorithm then the algorithm LARGERTOSMALLER runs in incremental-polynomial time. First, notice that the first for-loop takes only polynomial time in the size of the database. We can see easily that the time before printing the first graph is also bounded by a polynomial in the size of the database. Next, the for-loop on line 10 is repeated at most $|H|$-times for any graph $H$. Adding a graph $H^{\prime}$ to the data structure $A L L$ or getting occurrences of a graph $H^{\prime}$ from the data structure $A L L$ takes time polynomial in the number of graphs already stored in it and the size of the graph being stored (which is discussed in the main text). The number of graphs already stored in the data structure $A L L$ is bounded by $P \cdot M$ where $P$ is the number of already printed graphs and $M$ is the maximum size of a graph in the database. Thus, we have that the time between printing two consecutive graphs is bounded by a polynomial in the size of the database and in the number of already printed graphs, i.e. the algorithm runs in incremental polynomial time.

Fourth, we can show using essentially the same reasoning that if there is a polynomial-time graph canonization algorithm then the algorithm runs with polynomial delay.

Using the results on complexity of graph canonization for planar [12] and bounded-treewidth graphs [7], we can get the following corollary.

Corollary 4. The problem $\mathrm{ALL}_{L \rightarrow S}$ can be solved with polynomial delay for the classes of planar and bounded-treewidth graphs.

In fact, there are many other classes of graphs for which graph isomorphism is known to be decidable in polynomial time, e.g. graphs of bounded degree [8]. One can easily use the theorems presented in this section for such classes too as long as they are hereditary, as is the case for bounded-degree graphs.

\subsection{Other Negative Results}

The following theorem asserts that the results for the problem $\mathrm{ALL}_{L \rightarrow S}$ are essentially optimal in the sense that existence of a polynomial-time algorithm for 
graph isomorphism is both sufficient and necessary for existence of an incrementalpolynomial-time algorithm for the problem $\mathrm{ALL}_{L \rightarrow S}$.

Theorem 6. The problem $\mathrm{ALL}_{L \rightarrow S}$ can be solved in incremental-polynomial time for graphs from a hereditary class $\mathcal{G}$ if and only if graph isomorphism can be decided in polynomial-time for graphs from $\mathcal{G}$.

Proof. $(\Rightarrow)$ The proof idea is similar to the idea of the proof of Theorem 3. Let $H$ and $G$ be graphs from $\mathcal{G}$. We will show how to use an incrementalpolynomial-time algorithm for the problem $\operatorname{ALL}_{L \rightarrow S}$ to decide whether $H$ and $G$ are isomorphic in polynomial time. If $|V(H)| \neq|V(G)|$ or $|E(H) \neq| E(G) \mid$, we return 'not isomorphic'. Otherwise, we construct a database of graphs $D B=$ $\{H, G\}$ and let the algorithm for the problem $\operatorname{ALL}_{L \rightarrow S}$ run until it outputs a graph and its occurrences (implicitly giving us also the frequency) and then we stop it (it follows from definition of incremental-polynomial time that this will run only for time polynomial in the sizes of $H$ and $G$ ). Then the output graph has frequency 1 if and only if $H$ and $G$ are not isomorphic. So, if the frequency of the output graph is 1 we return 'not isomorphic' and if the frequency of the output graph is greater than 1 then we return 'isomorphic'. This gives us an algorithm for deciding isomorphism of graphs which runs in polynomial time.

$(\Leftarrow)$ This direction is explicitly shown in Theorem 5 .

The next theorem indicates that $\mathrm{ALL}_{L \rightarrow S}$ is the simplest (complexity-wise) from the enumeration problems considered in this paper because e.g. the problems $\mathrm{ALL}_{F \rightarrow I}$ and $\mathrm{ALL}_{I \rightarrow F}$ may be unsolvable in incremental-polynomial time even if a polynomial-time graph isomorphism algorithm existed.

Theorem 7. If $\mathrm{Gl} \in \mathrm{P}$ and $\mathrm{P} \neq \mathrm{NP}$ was true then there would be an incrementalpolynomial-time algorithm for the problem $\mathrm{ALL}_{L \rightarrow S}$ for the class $\mathcal{G}$ of all graphs but no incremental-polynomial-time algorithm for the problems $\mathrm{ALL}_{I \rightarrow F}$ and $\mathrm{ALL}_{F \rightarrow I}$ for the class of all graphs.

Proof. The positive result for the problem $\mathrm{ALL}_{L \rightarrow S}$ follows from Theorem 5 . The hardness of FCGM and FCIGM has been shown in [5] and in [4] using reductions from Hamiltonian cycle problem (for mining under subgraph isomorphism) and from maximum clique problem (for mining under induced subgraph isomorphism), from which the hardness result for the $\mathrm{ALL}_{F \rightarrow I}$ problem follows. The hardness of the $\mathrm{ALL}_{I \rightarrow F}$ problem follows from Theorem 3 .

For now, we leave open the question of complexity of the $\mathrm{ALL}_{S \rightarrow L}$ problem conditioned only on the pattern matching operator not being in P. Theorem 1 asserts that solving this problem in incremental polynomial time is not possible if the pattern matching operator is not fixed-parameter tractable with the parameter being the size of the pattern graph, which is a widely believed conjecture. 


\section{Mining under Homeomorphism and Minor Embedding}

Many of the results presented in this paper may be generalized to mining with other important pattern matching operators: (induced) homeomorphism and (induced) minor embedding. In this section, we briefly discuss these generalizations. Here, we only consider mining from unlabeled graphs because there is no generally agreed-upon definition of homeomorphism or minor embedding of labeled graphs for pattern mining ${ }^{10}$.

The ideas from Theorem 1 are not relevant for mining under minor embedding or homeomorphism because minor embedding and homeomorphism are fixedparameter tractable with the parameter being the size of the pattern graph as shown in $[11,3]$. However, it can be used together with the following theorem to show hardness of the $\mathrm{ALL}_{S \rightarrow L}$ problem under induced homeomorphism and induced minor embedding.

Theorem 8. Deciding induced homeomorphism or induced minor embedding $G_{1} \preccurlyeq G_{2}$ is not fixed-parameter tractable with the size of $G_{1}$ as the parameter (unless FPT $=W[1]$ ).

Proof. This can be shown by reduction from the $k$-independent set problem parameterized by $k$ which follows from the following simple observation. Let $G$ be a graph. $G$ contains an independent set of size $k$ if and only if $H \preccurlyeq G$ where $H$ is a graph consisting of $k$ isolated vertices. Notice that this theorem holds also when we restrict $G_{1}$ and $G_{2}$ to be connected graphs. The basic idea of the proof is then the same. The reduction from $k$-independent set problem is then as follows. We create a new graph $G^{\prime}$ by taking the graph $G$ from the proof, adding a new vertex and connecting it to all vertices of $G$. Instead of taking $H$ as a set of $k$ isolated vertices we let $H$ be a star on $k+1$ vertices (i.e. a tree in which all vertices are connected to one vertex $v$ ). We then again have $H \preccurlyeq G^{\prime}$ if and only if $G$ contains an independent set of size $k$.

Ideas analogical to those from Theorems 1, 2, 3 and 8 can be used to obtain the following negative results for mining under (induced) homeomorphism and (induced) minor embedding.

Theorem 9. The problem $\mathrm{ALL}_{S \rightarrow L}$ under induced homeomorphism or induced minor embedding cannot be solved in incremental-polynomial time for the class of all graphs (unless FPT $=W[1]$ ). The problems $\mathrm{ALL}_{F \rightarrow I}$ under (induced) homeomorphism or (induced) minor embedding cannot be solved in incrementalpolynomial time for the class of all graphs (unless $P=N P$ ). The problem $\mathrm{ALL}_{I \rightarrow F}$ under (induced) homeomorphism or (induced) minor embedding cannot be solved in incremental-polynomial time for the class of bounded-treewidth graphs (unless $P=N P$ ).

${ }^{10}$ For homeomorphism, for instance, we could allow the subdivided edges to have different labels or, to the contrary, we could require them all to have the same label etc. Then another question could be how we should treat labels of vertices etc. While these considerations are interesting even for practice, they are out of the scope of this paper. 
The positive results from Section 4.2 may be adapted for mining under (induced) homeomorphism and (induced) minor embedding as follows. We can essentially use the algorithm LARGERTOSMALLER as is, but we need to modify the procedure REFINE $(H)$ (the modified algorithm will be denoted as LARGERToSMALLER $^{*}$ to avoid confusion). For mining under minor embedding, when given a graph $H$, the procedure REFINE should return all graphs $H^{\prime}$ which can be obtained from $H$ by removing an edge or by contracting an edge and removing loops and multiple edges thus produced. For mining under homeomorphism, it should return all graphs $H^{\prime}$ which can be obtained from $H$ by removing an edge or by contracting an edge incident to a vertex of degree 2. For mining under induced minor embedding, it should return all graphs $H^{\prime}$ which can be obtained from $H$ by removing a vertex and all its incident edges or by contracting an edge and removing loops and multiple edges thus produced. Finally, for mining under induced homeomorphism, it should return all graphs $H^{\prime}$ which can be obtained from $H$ by removing a vertex and all its incident edges or by contracting an edge incident to a vertex of degree 2 .

Theorem 10. Let $\mathcal{G}$ be a class of graphs closed under formation of minors admitting a polynomial-time isomorphism algorithm and let the pattern matching operator $\preccurlyeq$ be either (induced) homeomorphism of (induced) minor embedding. Given a database $D B$ of graphs from $\mathcal{G}$, the algorithm LARGERTOSMALLER* solves the problem $\mathrm{ALL}_{L \rightarrow S}$ in incremental polynomial time. If the graphs from $\mathcal{G}$ also admit a poly-time canonization then the algorithm LARGERTOSMALLER* solves the problem $\mathrm{ALL}_{L \rightarrow S}$ with polynomial delay.

Proof (Sketch). We can essentially use the reasoning from the proof of Theorem 5 . We only need to notice that $\preccurlyeq$ is transitive and that if $H \preccurlyeq G$ then a graph isomorphic to $H$ can be obtained from $G$ by a repeated application of the procedure REFINE. For instance, to show that all graphs homeomorphic to at least one database graph will be printed eventually, we can reason as follows. We can show using Theorem 5 that every graph which is subgraph isomorphic to at least one database graph must also be printed by the algorithm LARGERTOSMALLER* If a graph $H$ is homeomorphic to a database graph $G$ then $G$ has a subgraph $G^{\prime}$, which must be printed at some point, which is isomorphic to a graph which can be obtained from $H$ by subdividing its edges. But this also means that $H$ can be obtained from $G^{\prime}$ by repeatedly contracting some of its edges incident to a vertex of degree 2 and removing the loops produced by this process. Thus, any graph homeomorphic to at least one of the database graphs will be printed eventually. It is also not difficult to see that all graphs produced by the refinement function REFINE must be homeomorphic to the graph being refined. Since homeomorphism is transitive any of the produced graphs will be homeomorphic to at least one of the database graphs. Finally, to show that the occurrences printed for every output graph are correct, we can repeat the reasoning from Theorem 5 (which we omit here due to space constraints). 
Using the fact that bounded-treewidth and planar graphs are closed under formation of graph minors and that they also admit a polynomial-time isomorphism algorithm, we can obtain the following corollary.

Corollary 5. The problem $\mathrm{ALL}_{L \rightarrow S}$ under (induced) homeomorphism or (induced) minor embedding can be solved with polynomial delay for the classes of planar and bounded-treewidth graphs.

\section{Conclusions and Future Work}

In this paper, we have shown how different orders in which graphs are enumerated affect computational complexity of the mining problem. We have presented several negative results. We have also described a positive result which shows that it is possible to mine all graphs from a database of bounded-treewidth or planar graphs with polynomial delay under any of the following six different pattern matching operators: (induced) isomorphism, (induced) homeomorphism and (induced) minor embedding. Here, by mining all graphs, we mean enumerating all graphs which have frequency at least one and printing their occurrences in the database. This result holds despite the fact that deciding any of the six pattern matching operators is NP-hard. However, since the positive result depends heavily on mining all graphs and not just the frequent ones, the question whether frequent graph mining is achievable with polynomial delay for an NPhard pattern matching operator, remains open. In fact, as we have shown, e.g. for planar graphs the latter problem of mining frequent graphs cannot be done even in incremental polynomial time, whereas mining all graphs can be done with polynomial delay.

Lastly, it is worth noting that when we performed preliminary experiments with a simple implementation of the algorithm for the $A L L_{L \rightarrow S}$ problem, we were able to mine completely about $70 \%$ molecules from NCI GI dataset consisting of approximately three and half thousand organic molecules. This suggests that the techniques presented in this paper might also lead to development of practical graph mining algorithms. For instance, it would not be difficult to obtain a graph mining algorithm, having similar positive complexity guarantees as the LARGERTOSMALLER algorithm, for mining graphs of bounded radius (from database graphs of arbitrary radius) or with constraints on minimum vertex degree ${ }^{11}$ etc. Exploring these and similar ideas is left for future work.

Acknowledgement. This work has been supported by ERC Starting Grant 240186 "MiGraNT: Mining Graphs and Networks, a Theory-based approach".

11 The latter constraint on degrees would not probably be very relevant for boundedtreewidth graphs or planar graphs. This is because any graph of treewidth $k$ must always have at least one vertex of degree at most $k$ and because any planar graph must always have at least one vertex of degree at most 5. However, isomorphism algorithms are extremely fast in practice, despite not being polynomial in the worst case, so the algorithm that we would obtain for general graphs could still be practical. 
The first author is supported by a grant from the Leverhulme Trust (RPG-2014164).

\section{References}

1. J. Flum and M. Grohe. Parameterized Complexity Theory. Berlin: Springer Verlag, 2006.

2. M. R. Garey, D. S. Johnson, and R. E. Tarjan. The planar hamiltonian circuit problem is np-complete. SIAM J. Comput., 5(4):704-714, 1976.

3. M. Grohe and D. Marx. Structure theorem and isomorphism test for graphs with excluded topological subgraphs. CoRR, abs/1111.1109, 2011.

4. T. Horváth, K. Otaki, and J. Ramon. Efficient frequent connected induced subgraph mining in graphs of bounded tree-width. In Machine Learning and Knowledge Discovery in Databases - European Conference, ECML PKDD 2013, pages 622-637, 2013.

5. T. Horváth and J. Ramon. Efficient frequent connected subgraph mining in graphs of bounded tree-width. Theor. Comput. Sci., 411(31-33):2784-2797, 2010.

6. D. S. Johnson, M. Yannakakis, and C. H. Papadimitriou. On generating all maximal independent sets. Information Processing Letters, 27(3):119 - 123, 1988.

7. D. Lokshtanov, M. Pilipczuk, M. Pilipczuk, and S. Saurabh. Fixed-parameter tractable canonization and isomorphism test for graphs of bounded treewidth. In 55th IEEE Annual Symposium on Foundations of Computer Science, FOCS 2014, pages 186-195, 2014.

8. E. M. Luks. Isomorphism of graphs of bounded valence can be tested in polynomial time. Journal of Computer and System Sciences, 25(1):42 - 65, 1982.

9. D. Marx and M. Pilipczuk. Everything you always wanted to know about the parameterized complexity of subgraph isomorphism (but were afraid to ask). In 31st International Symposium on Theoretical Aspects of Computer Science (STACS 2014), STACS 2014, pages 542-553, 2014.

10. J. Matoušek and R. Thomas. On the complexity of finding iso- and other morphisms for partial k-trees. Discrete Mathematics, 108(1-3):343-364, 1992.

11. N. Robertson and P. D. Seymour. Graph minors .xiii. the disjoint paths problem. J. Comb. Theory, Ser. B, 63(1):65-110, 1995.

12. J. Torán and F. Wagner. The complexity of planar graph isomorphism. Bulletin of the EATCS, 97:60-82, 2009. 